\title{
The Discounted Payback in Investment Appraisal: A Case Study
}

\author{
Samih Antoine Azar ${ }^{1} \&$ Nazim Noueihed ${ }^{2}$ \\ ${ }^{1}$ Faculty of Business Administration \& Economics, Haigazian University, Lebanon \\ ${ }^{2}$ Department of Mathematics, Division of Mathematical Sciences, Faculty of Arts \& Sciences, Haigazian University, \\ Lebanon \\ Correspondence: Samih Antoine Azar, Professor, Faculty of Business Administration \& Economics, Haigazian \\ University, Mexique Street, Kantari, Beirut, Lebanon. Tel: 96-1134-9230. E-mail: samih.azar@haigazian.edu.lb
}

Received: August 22, 2014

Accepted: September 3, 2014

Online Published: September 5, 2014

doi:10.5430/ijba.v5n5p58

URL: http://dx.doi.org/10.5430/ijba.v5n5p58

\begin{abstract}
The purpose of this paper is to study a Monte Carlo simulation of the discounted payback, and its application to investment appraisal. The underlying project in the case study has a useful life of 10 years with an initial outlay of $\$ 2,000$, and with stochastic, independent, and normally distributed cash inflows. These cash inflows are simulated from the same normal frequency distribution, i.e. from a distribution with the same average of $\$ 400$ and standard deviation of $\$ 80$. The number of Monte Carlo simulation runs is set at 300 . The empirical analysis presents descriptive statistics of the nine parameters of concern in the simulations. It also tests for normality of these nine generated series. In addition, it carries out simple hypothesis testing. In most cases the theoretical values are known in advance and testing makes sure that these expectations are statistically met by the simulations. A model of the discounted payback, in terms of the average cash flow, is derived and is estimated. The model is tested by non-linear regression analysis with great success. The final part of the empirical analysis assesses the information content of the discounted payback in predicting the net present value and compares it with the explanatory power of the internal rate of return. The paper concludes by highlighting the importance and usefulness of the discounted payback despite its many detractors.
\end{abstract}

Keywords: investment appraisal, payback, discounted payback, net present value, internal rate of return, case study, stochastic cash flows, Monte Carlo simulation, hypothesis testing, non-linear regression, information content

\section{Introduction}

There is more than one method of investment appraisal: the benefit-cost analysis, the profitability index (PI), the average book rate of return (ARR), the payback (PP), the discounted payback (DPP), the internal rate of return (IRR), and the net present value (NPV). Benefit-cost analysis is mostly used in public finance and features elements related to the NPV rule. The profitability index is operational and convenient when there is capital rationing. According to Graham and Harvey (2001) 75\% of CFOs adopt the NPV criterion, 76\% the IRR, and 57\% the payback. Brealey et al. (2014: 107) recognize that "firms often look at more than one measure of a project's attractiveness." They add that managers use the discounted payback as a "warning signal" for a bad investment (Brealey et al., 2104: 111). While the discounted payback rightfully takes into consideration the time value of money, it has the unwanted characteristic of ignoring cash flows that occur after the payback, which leads usually in wrongfully rejecting projects that are long term in nature. However, Bhandari (1986: 22) writes that "DPP is closely related to NPV, IRR, and PI. The only limitation DPP has is that it ignores cash flows beyond the DPP, but it does so only after assuring the profitability of the project. For small businesses and venture capitalists who are interested in maximum liquidity (quickest recovery of capital outlay) along with specification of a minimum required profitability, the DPP is a better decision-making criterion than PP, NPV, ARR, IRR, and PI." Bhandari (1986) therefore stresses the issue of liquidity in favoring the discounted payback as a criterion in determining project feasibility. It is maybe not surprising that references to research on the discounted payback are so rare. It seems that the literature has already decided against the discounted payback (DPP) both as a practical tool and as an academic construct. The purpose of this paper is to restore to the DPP part of its previous popularity, glory, and notoriety.

The paper is organized as follows. The next section is the empirical analysis. It has 11 subsections. The first subsection summarizes the simulation model which is a case study of a 10 -year project with a given initial outlay, 
and with stochastic, independent, and normally distributed cash inflows. These cash inflows are simulated from the same normal frequency distribution, i.e. from a distribution with the same average and standard deviation. The number of simulation runs is set at 300 . The second subsection provides for the descriptive statistics of the nine parameters of concern. The third subsection tests for normality of these nine generated series. Subsection 4 to subsection 9 carry out simple hypothesis testing. In most cases the theoretical values are known in advance and testing makes sure that these expectations are statistically met by the Monte Carlo simulations. Subsection 10 provides for a model of the discounted payback (DPP) in terms of the average cash flow. The model is tested by non-linear regression analysis with paramount success. The final subsection determines the information content of the discounted payback in predicting the NPV and compares it with the explanatory power of the IRR. The final section summarizes and concludes.

\section{The Empirical Analysis}

The empirical analysis dwells mainly on hypothesis testing of key parameters. The Monte Carlo simulation model is introduced in the next subsection. In all nine parameters are defined and generated. Then descriptive statistics and normality tests on the simulated parameters are presented. The subsequent six subsections carry out hypothesis testing on the central tendencies and dispersions of the simulated parameters. All tests are positive except for two. The latter are about tests on the mean and on the median of the discounted payback. The reason behind these test failures is later elucidated. Subsection 10 estimates a model of the discounted payback as a function of the average cash flow. The estimated coefficients in that function conform to theoretical reasoning. The last subsection unveils the information content of the discounted payback. While it is shown that the IRR explains $98.89 \%$ of the variation of the NPV, it is also shown that the discounted payback explains $85.44 \%$ of the same variation. This renders the discounted payback quite valuable in decision-making.

\subsection{The Model and the Simulation Parameters}

The model assumes a project with an initial cash outlay fixed at $\$ 2,000$ and a useful life of 10 years. Each yearly cash flow is independent of the other and is simulated to follow a normal distribution with mean $\$ 400$ and standard deviation $\$ 80$. The standard deviation is chosen to be much lower than the mean in order to prevent and avoid negative generated cash flows. The discount rate is set at $10 \%$. In all there are nine parameters in each simulation run. The first two are the payback and the discounted payback. Then the NPV and the IRR are calculated. After that the average, minimum, maximum, standard deviation and variance of the 10 yearly cash flows are computed. The Monte Carlo simulation is repeated 300 times, which means that 300 estimates of each parameter are generated. Two simulations, in addition to the 300 , provided for a negative NPV and were omitted from the analysis. This is because a negative NPV implies a payback larger than the useful life of the project.

\subsection{The Descriptive Statistics}

Table 1 summarizes the descriptive statistics on the nine generated series. In the table the following items are calculated for each series: the mean, the median, the maximum, the minimum, and the standard deviation. The number of simulations, and therefore the sample size, is 300.The mean is slightly higher than the median for the payback, for the NPV, for the minimum, for the maximum, for the standard deviation, and for the variance. The mean is slightly lower than the median for the remaining three series: the discounted payback, the IRR, and the average cash flow variable. The latter is calculated by taking the average of the ten yearly cash flows for each simulation run. The volatility of the payback (0.45067) is lower than that of the discounted payback (0.79194). The NPV is positive and ranges widely between $\$ 97.64$ and $\$ 914.67$. The minimum IRR (11.1073\%) is higher than the selected discount rate of $10 \%$. This is as expected because the NPVs are all positive. The 300 averages of the ten yearly cash flows fluctuate between $\$ 332.739$ and $\$ 465.64$, and their grand mean is close to the simulated mean of $\$ 400$ (\$397.761). The maximum of the 300 minima yearly cash flows is slightly lower than $\$ 400(\$ 388.067)$. The minimum of the 300 maxima yearly cash flows is slightly higher than $\$ 400$ (\$401.348). The 300 standard deviations of the NPVs vary widely between $\$ 31.467$ and $\$ 145.616$ around a mean of $\$ 77.906$, which is close to the simulated standard deviation of $\$ 80$. The 300 variances vary widely between 990.19 and 21,204.08 around a mean of 6453.92, which is close to the simulated variance of 6400 . 
Table 1. Descriptive statistics on the nine simulated parameters

\begin{tabular}{|c|c|c|c|c|c|c|c|c|c|}
\hline Variable & Payback & $\begin{array}{c}\text { Discounted } \\
\text { payback }\end{array}$ & NPV & IRR & $\begin{array}{l}\text { Average } \\
\text { cash flow }\end{array}$ & Min & Max & $\begin{array}{l}\text { Std. } \\
\text { dev. }\end{array}$ & Variance \\
\hline Mean & 5.04941 & 7.10823 & 443.206 & 14.93724 & 397.761 & 272.064 & 517.626 & 77.906 & 6453.92 \\
\hline Median & 5.02582 & 7.14093 & 443.065 & 14.95295 & 398.379 & 270.999 & 513.549 & 77.218 & 5962.58 \\
\hline Maximum & 6.22142 & 9.09865 & 914.670 & 20.22460 & 465.647 & 388.067 & 666.929 & 145.616 & 21204.08 \\
\hline Minimum & 3.94381 & 5.39116 & 97.640 & 11.10730 & 332.739 & 116.403 & 401.348 & 31.467 & 990.190 \\
\hline Std. dev. & 0.45067 & 0.79194 & 160.030 & 1.787513 & 24.973 & 48.808 & 46.722 & 19.645 & 3254.35 \\
\hline $\mathrm{N}$ & 300 & 300 & 300 & 300 & 300 & 300 & 300 & 300 & 300 \\
\hline
\end{tabular}

The short phrase "Std dev." stands for the standard deviation. $\mathrm{N}$ is the number of simulation runs.

\subsection{The Normality of the Statistics}

Table 2 presents the normality tests on the nine key variables. There are five different tests and these are enumerated in the first column of Table 2. Most of the tests are concordant although there are notable differences like for the discounted payback and the maximum cash flow variable for every run. Later it is shown that the discounted payback is indeed very special as its frequency distribution is made out of clusters (see Figure 1 below). There is no theoretical reason for the nine variables to be normally distributed. However if the central limit theorem is invoked and since the sample size is relatively large, consisting of 300 data points for each variable, asymptotic normality can be assumed. One problematic finding is the non-normality of the variance variable at a time when the standard deviation variable is seen to be normally distributed. Finally, it is surprising that the minimum cash flow per run is distributed normally, and that the maximum cash flow for each run is very close to be normally distributed with only two tests out of the five rejecting normality.

Table 2. Normality tests. Actual p-values are reported under the null hypothesis of normality

\begin{tabular}{|c|c|c|c|c|c|c|c|c|c|}
\hline Test & Payback & $\begin{array}{c}\text { Discounted } \\
\text { payback }\end{array}$ & NPV & IRR & $\begin{array}{l}\text { Average } \\
\text { cash flow }\end{array}$ & Min & Max & $\begin{array}{l}\text { Std. } \\
\text { dev. }\end{array}$ & Variance \\
\hline Lilliefors (D) & $>0.1$ & 0.0000 & $>0.1$ & $>0.1$ & $>0.1$ & $>0.1$ & $>0.1$ & $>0.1$ & 0.0000 \\
\hline \multicolumn{10}{|l|}{ Cramer-von } \\
\hline Mises (W2) & 0.1070 & 0.0000 & 0.1766 & 0.1068 & 0.9357 & 0.1760 & 0.0512 & 0.1752 & 0.0000 \\
\hline Watson (U2) & 0.1061 & 0.0000 & 0.1521 & 0.0903 & 0.9251 & 0.2185 & 0.1293 & 0.3236 & 0.0000 \\
\hline \multicolumn{10}{|c|}{ Anderson-Darling } \\
\hline (A2) & 0.1463 & 0.0000 & 0.1663 & 0.1073 & 0.8534 & 0.0844 & 0.0269 & 0.0618 & 0.0000 \\
\hline Jarque-Bera & 0.1750 & 0.0640 & 0.1734 & 0.1330 & 0.4002 & 0.0882 & 0.0055 & 0.0216 & 0.0000 \\
\hline
\end{tabular}

The short phrase "Std dev." stands for the standard deviation.

\subsection{The Mean and Median of the NPVS}

The theoretical mean NPV is calculated by discounting the ten yearly cash flows of $\$ 400$ to the present with a $10 \%$ discount rate and subtracting the initial cash outlay of $\$ 2,000$. As a result the mean NPV is expected to be $\$ 457.827$. The simulated mean NPV is $\$ 443.2055$. A hypothesis test using the sample standard deviation produces a t-statistic of -1.582507 that has a two-tailed actual p-value of 0.1146, failing to reject the null hypothesis that the sample mean is equal to the theoretical mean. A hypothesis test using the theoretical standard deviation, obtained below in the following subsection, produces a z-statistic of -1.572211 that has a two-tailed actual p-value of 0.1159 , failing to reject the null hypothesis that the sample mean is equal to the theoretical mean. Therefore the simulation results are sound.

The sample median for the NPVs is $\$ 443.065$. The null hypothesis that this sample median is equal to the theoretical mean of $\$ 457.827$ fails to be rejected with actual p-values ranging between 0.0984 and 0.3865 . Four tests are carried 
out: the sign test with a binomial distribution, the sign test with the normal approximation to the binominal, the Wilcoxon signed rank test, and the van der Waerden (normal scores) test.

\subsection{The Variance of the NPVS}

The theoretical variance of the 300 simulated NPVs can be obtained from the NPV equation:

$$
N P V=-2,000+\sum_{t=1}^{10} \frac{C_{t}}{(1+0.1)^{t}} \Rightarrow \text { Variance }(N P V)=\text { Variance }\left(C_{t}\right)\left[\sum_{t=1}^{10} \frac{1}{(1+0.1)^{2 t}}\right]=25,946.0989
$$

In this equation the two facts that the cash flows are independent and that Variance $\left(C_{t}\right)=80^{2}=6400$ are used (see subsection 2.1 above). A hypothesis test that the actual variance, which equals $25,609.59$, is equal to the theoretical variance gives an actual two-tailed p-value of 0.4475 , failing to reject the null hypothesis. The simulation results are here also sound.

\subsection{The Mean and the Median of the IRRs}

The theoretical mean IRR $(r)$ is obtained by solving the following equation for $r$ :

$$
0=-2,000+\sum_{t=1}^{10} \frac{400}{(1+r)^{t}} \Rightarrow r=15.098414 \%
$$

A hypothesis test that the actual mean IRR, which equals $14.93724 \%$, is statistically insignificantly different from the above theoretical mean IRR gives a t-statistic of -1.561752 which has an actual two-tailed p-value of 0.1194 , failing to reject the null hypothesis of equality. Here again the simulation results are sound.

The sample median IRR is $14.95295 \%$. The null hypothesis that this sample median is equal to the mean theoretical IRR fails to be rejected with actual p-values ranging between 0.1028 and 0.1842 . Here gain the same four tests as in subsection 2.4 are carried out.

\subsection{The Mean and Median of the Paybacks}

The theoretical payback is $2000 / 400=5$ years (see Marshall, 1985). The actual mean payback from the 300 simulations is 5.049405 years. A test that this value is statistically insignificantly different from 5 gives a t-statistic of 1.898771 that has an actual two-tailed p-value of 0.0586 failing to reject the null of equality. Hence the simulation results are sound.

The sample median payback is 5.025821 years. The null hypothesis that this sample median is equal to the expected theoretical mean of 5 years fails to be rejected with actual p-values ranging between 0.0511 and 0.6034 . The same four tests as in section 2.4 are carried out.

\subsection{The Mean and Median of the Discounted Paybacks}

The theoretical mean of the discounted payback is obtained by solving the following equation for $y$ :

$$
2,000=\sum_{t=1}^{y} \frac{C_{t}}{(1+0.1)^{t}}=\sum_{t=1}^{y} \frac{400}{(1+0.1)^{t}} \Rightarrow y=7.282056 \text { years }
$$

The actual sample mean is 7.108229 years. Although the sample mean is close to the theoretical mean the null hypothesis that they are equal to each other is rejected with a t-statistic of -3.801781 that has an actual two-tailed $\mathrm{p}$-value of 0.0002 , rejecting equality at very low marginal significance levels. The reason for the rejection may be due to the fact that, unlike all the rest eight parameters, the sample frequency distribution of the discounted payback is made out of clusters for certain given values. See the attached histogram (Figure 1). The reason for the clusters is unknown. However, as the discount rate is reduced the clusters tend to disappear. So it is not due to the underlying formulae used for the discounted payback.

The sample median discounted payback is equal to 7.140931 years. The null hypothesis that this sample median is equal to the expected theoretical mean of 7.282056 years is rejected with actual p-values less than 0.0001 for the four tests carried out as in section 2.4. The reason for this rejection may be due to the clusters in the frequency distribution of the discounted payback (see Figure 1). 


\subsection{The Means of the Standard Deviations and the Variances}

The theoretical standard deviation is $\$ 80$. The simulated standard deviation is the standard deviation of the 10 simulated cash flows. Therefore there are 300 simulated standard deviations. The sample mean standard deviation is $\$ 77.90554$. The null hypothesis that this value is statistically insignificantly different from the theoretical value of $\$ 80$ gives a t-statistic of -1.846616 that has an actual two-tailed p-value of 0.0658 , failing to reject the null of equality. A test that the actual mean variance, which is 6453.921 , is statistically insignificantly different from the theoretical square of 80 , or 6400 , fails to reject the hypothesis of equality with an actual t-statistic of 0.286980 that has an actual two-tailed p-value of 0.7743 . Once again the simulation results are sound.

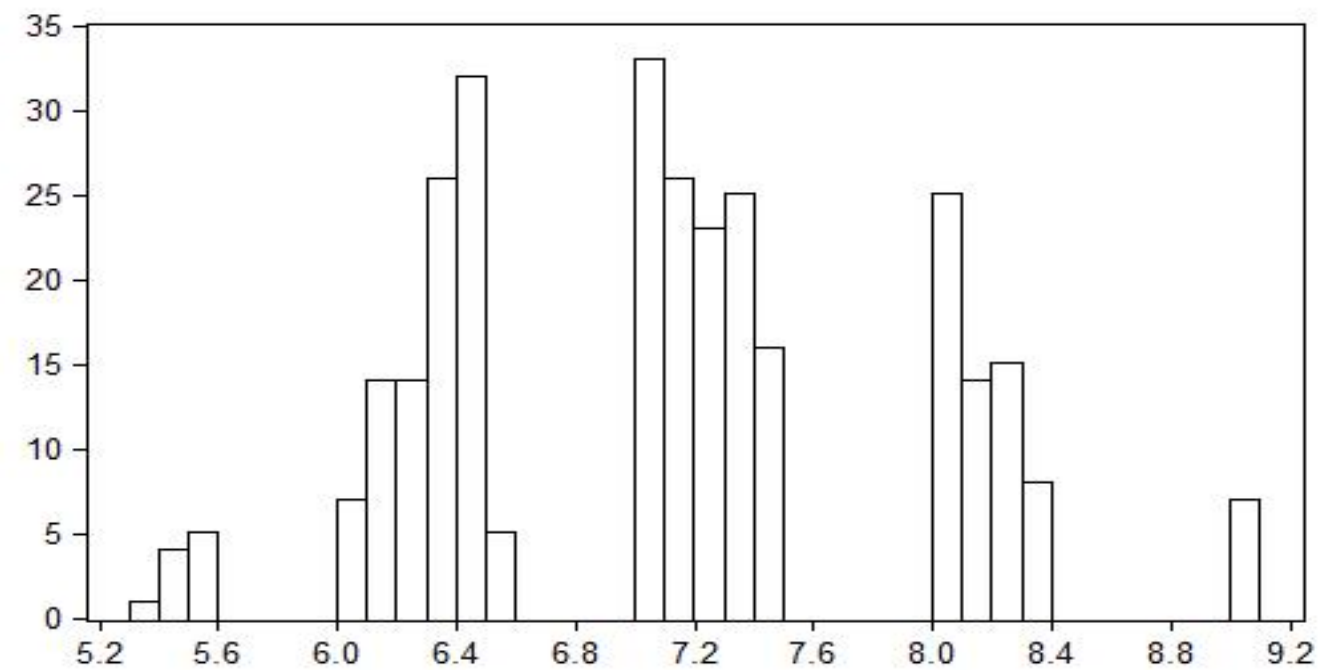

Figure 1. Frequency distribution of the discounted payback

\subsection{The Fit and Econometric Diagnostics of the Discounted Paybacks}

The equation for the discounted payback is repeated here as stated previously in subsection 2.8, with $i$ being the discount rate, and the following equations are deduced (see also Marshall, 1985):

$$
2,000=\sum_{t=1}^{y} \frac{C_{t}}{(1+i)^{t}} \Rightarrow 2,000=E\left(C_{t}\right) \sum_{t=1}^{y} \frac{1}{(1+i)^{t}} \Rightarrow y=D P P=\frac{-1}{\log (1+i)} \log \left(1-\frac{2,000}{E\left(C_{t}\right)} i\right)
$$

In this final equation the variable $E\left(C_{t}\right)$, with $E$ being the expectation operator, is what has been called previously as the average cash flow, i.e. the average of the ten generated cash flows in each simulation run. This final equation is a relation between the discounted payback, $y$ or DPP, for each simulation run, and the average cash flow variable, again for each simulation run, and for a given discount rate $i$. Hence this final equation can be considered as a non-linear regression equation between those two variables. If one starts by setting the discount rate $i$ at $10 \%$, the following non-linear regression can be estimated:

$$
D P P=\alpha-\beta \frac{1}{\log (1+0.1))} \log \left(1-\frac{2,000 * 0.1}{E\left(C_{t}\right)}\right)+\varepsilon
$$

The parameters $\alpha$ and $\beta$ are coefficients to be estimated and $\varepsilon$ is the regression residual. Heteroscedasticity and autocorrelation consistent (HAC) and robust standard errors are computed (Newey and West, 1987). The estimate of the $\alpha$ coefficient is -0.113946 and carries a t-statistic of -0.464531 . Therefore, as expected, it is not statistically 
significantly different from zero. The estimate of the $\beta$ coefficient is 0.976475 and carries a t-statistic of 29.61905.The null hypothesis that this coefficient is statistically insignificantly different from +1 , as expected, fails to be rejected with a t-statistic of -0.713584 and an actual two-tailed p-value of 0.4760 . The adjusted R-square is 0.717334, which is relatively high. The Quandt-Andrews unknown breakpoint test, (Andrews, 1993; Andrews and Ploberger, 1994) with a total number of 211 breaks compared, fails to find any breakpoint at marginal significance levels higher than $20 \%$. The Ramsey RESET test fails to find instability and misspecification with an actual p-value of 0.4600 under the null of stability and correct specification. The residual $\varepsilon$ is found to be normally distributed by all tests introduced in subsection 2.3 above with the lowest actual p-value of 0.213801 under the null of normality.

The non-linear regression can be modeled as follows with the discount rate $i$ set to be estimated:

$$
D P P=\alpha-\frac{1}{\log (1+\beta)} \log \left(1-\frac{2,000 \beta}{E\left(C_{t}\right)}\right)+\varepsilon
$$

Again the parameters $\alpha$ and $\beta$ are coefficients to be estimated and $\varepsilon$ is the regression residual. Heteroscedasticity and autocorrelation consistent (HAC) and robust standard errors are computed (Newey and West, 1987). The estimate of the $\alpha$ coefficient is -0.203405 and carries a t-statistic of -1.80968 . Therefore, as expected, it is not statistically significantly different from zero. The estimate of the $\beta$ coefficient is 0.097701 and carries a $\mathrm{t}$-statistic of 32.90362 . The null hypothesis that this coefficient is statistically insignificantly different from $10 \%$, as expected, fails to be rejected with a t-statistic of -0.774116 and an actual two-tailed p-value of 0.4395 . The adjusted R-square is 0.717384 , which is relatively high. The residual $\varepsilon$ is found to be normally distributed by all tests introduced in subsection 2.3 above with the lowest actual p-value of 0.212548 under the null of normality.

\subsection{The Information Content of the Discounted Paybacks}

It is known that the NPV and the IRR of a given project should be closely related. This is true here also with the 300 simulated NPVs and IRRs. The regression of the NPVs on the IRRs gives a constant of -886.638 , with a t-statistic of -117.091 , and a slope of 89.02874, with a t-statistic of 168.9437. These t-statistics are calculated with robust heteroscedasticity and autocorrelation consistent (HAC) standard errors (Newey and West, 1987). The fit is near perfect with an adjusted R-square of 0.988871. This is as expected. However what is unexpected is the high explanatory power of the discounted payback in predicting the NPV. The regression of the NPVs on the discounted paybacks, with HAC robust standard errors, produces a constant of 1771.277, with a t-statistic of 57.9453, and a slope of -186.8358 , with a t-statistic of -44.71624 . The regression residuals are normally distributed, with an actual p-value of 0.4568 for the Jarque-Bera normality test, under the null of normality. In addition, the Quandt-Andrews unknown breakpoint test, for a number of 211 breaks compared, does not identify any break. The fit is quite strong with an adjusted R-square of 0.854379 . Therefore the information content of the discounted payback is literally huge. And the regression results are meaningful in a different way. These results show that reducing the discounted payback by one year increases the NPV by $\$ 186.8358$, a relation which can be used in managerial decision-making, or in ex post managerial reevaluation of the project.

\section{Conclusion}

The purpose of this paper is to study a Monte Carlo simulation of the discounted payback, and its application to investment appraisal. The underlying project in the case study has a useful life of 10 years with an initial outlay of $\$ 2,000$, and with independent, and normally distributed cash inflows, with the same average of $\$ 400$ and the same standard deviation of $\$ 80$. The number of Monte Carlo simulation runs is set at 300 . The empirical analysis includes descriptive statistics of the nine parameters of concern in the simulations. It also tests for normality of these nine generated series. In addition, it carries out simple hypothesis testing. In most cases the theoretical values are known in advance and testing makes sure that these expectations are statistically met by the simulations. A model of the discounted payback in terms of the average cash flow for each simulation run is derived and is estimated. The model is tested by non-linear regression analysis with great success. Finally, the information content of the discounted payback in predicting the net present value is assessed and a comparison with the explanatory power of the internal rate of return is provided. The conclusion is that the discounted payback is still highly important and useful for investment appraisal despite its many detractors.

\section{References}

Andrews, D. W. K. (1993). Tests for parameter instability and structural change with unknown change point. Econometrica, 61(4), 821-856. http://dx.doi.org/10.2307/2951764 
Andrews, D. W. K., \& Ploberger, W. (1994). Optimal tests when a nuisance parameter is present only under the alternative. Econometrica, 62(6), 1383-1414. http://dx.doi.org/10.2307/2951753

Bhandari, S. B. (1986). Discounted payback: A criterion for capital investment decisions. Journal of Small Business Management, 24(2), 16-22.

Brealey, R. A., Myers, S. C., \& Allen, F. (2014). Principles of corporate finance (1 $1^{\text {th }}$ global edition). New York, New York: McGraw-Hill.

Graham, J. R., \& Harvey, C. R. (2001). The theory and practice of finance: Evidence from the field. Journal of Financial Economics, 61, 187-203. http://dx.doi.org/10.1016/S0304-405X(01)00044-7

Marshall, H. E. (1985). A graphical approach to discounted payback. Construction Management and Economics, 3, 105-120. http://dx.doi.org/10.1080/01446198500000009

Newey, W., \& West, K. (1987). A simple positive semi-definite, heteroskedasticity and autocorrelation consistent covariance matrix. Econometrica, 55, 703-708. http://dx.doi.org/10.2307/1913610 